Marquette University

e-Publications@Marquette

Fall 2018

\title{
Comparing procedures on the acquisition and generalization of tacts for children with autism spectrum disorder
}

Norma R. Schnell

Marquette University

Jason C. Vladescu

Caldwell University

Tiffany Kodak

Marquette University, tiffany.kodak@marquette.edu

Casey L. Nottingham

Caldwell Univesity

Follow this and additional works at: https://epublications.marquette.edu/psych_fac

Part of the Psychology Commons

\section{Recommended Citation}

Schnell, Norma R.; Vladescu, Jason C.; Kodak, Tiffany; and Nottingham, Casey L., "Comparing procedures on the acquisition and generalization of tacts for children with autism spectrum disorder" (2018).

Psychology Faculty Research and Publications. 438.

https://epublications.marquette.edu/psych_fac/438 


\section{Marquette University}

\section{e-Publications@Marquette}

\section{Psychology Faculty Research and Publications/College of Arts and Sciences}

This paper is NOT THE PUBLISHED VERSION; but the author's final, peer-reviewed manuscript. The published version may be accessed by following the link in the citation below.

Journal of Applied Behavior Analysis, Vol. 51, No. 4 (Fall 2018): 769-783. DOI. This article is (C) Wiley and permission has been granted for this version to appear in e-Publications@Marquette. Wiley does not grant permission for this article to be further copied/distributed or hosted elsewhere without the express permission from Wiley.

\section{Comparing procedures on the acquisition and generalization of tacts for children with autism spectrum disorder}

Lauren Schnell: Caldwell University

Jason C. Vladescu: Caldwell University

Tiffany Kodak: University of Wisconsin Milwaukee

Casey L. Nottingham: Caldwell University

\section{Abstract}

Generalization is a critical outcome for individuals with autism spectrum disorder (ASD) who display new skills in a limited range of contexts. In the absence of proper planning, generalization may not be observed. The purpose of the current study was to directly compare serial to concurrent multiple exemplar training using total training time per exemplar, mean total training time, and exposures to mastery across three children diagnosed with ASD. Additionally, we assessed the efficiency of presenting secondary targets in the antecedent and consequence portions of learning trials and evaluated generalization to tacts not associated with direct teaching. Results suggested that all training conditions produced acquisition and generalization for trained and untrained exemplars. However, the serial multiple exemplar training condition was more efficient for two participants, 
whereas the instructive feedback condition was the most efficient for the third. Findings are discussed considering previous studies and areas for future research.

Stimulus generalization is the spread of the effects of reinforcement from one stimulus condition to another, without direct training (Catania, 2007), and is considered a defining characteristic and a fundamental concern of applied behavior analysis (Baer, Wolf \& Risley, 1968; Stokes \& Baer, 1977). Because individuals with autism spectrum disorder (ASD) may display new skills in a limited range of contexts, proper planning is typically required for these skills to generalize (Rimland, 1964). One recommendation to achieve generalization is to train sufficient exemplars of stimuli within a class (Stokes \& Baer, 1977).

Two approaches for doing so are serial and concurrent multiple exemplar training (MET). In serial MET (S-MET), training is introduced for one training exemplar at a time, and tests are conducted to determine if responding generalizes to several (e.g., three) generalization exemplars (Schroeder \& Baer, 1972). S-MET has the potential advantage of being relatively easy to implement, as instructors need present only one exemplar per training target in a sequential fashion. For example, Eikeseth and Nesset (2003) observed acquisition and generalized responding using S-MET to teach vocal imitation of letter sound blends to two participants, ages 6 and 5 years old, with phonological disorders. Participants were taught a specific letter sound blend (e.g., /sk/) using one Norwegian word (e.g., skap), then probes were conducted to determine if participants would demonstrate generalized responding of the letter sound blend to other Norwegian words containing that same sound (e.g., sko, skatt, etc.). If generalized responding was not observed, the experimenters trained a second exemplar (i.e., another Norwegian word containing the specific letter sound blend). Both participants emitted the letter sound blend for targeted words and demonstrated generalized responding to untargeted words. The number of trained exemplars varied for each letter sound blend and participant, ranging from one to eight.

In concurrent MET (C-MET), training is introduced for multiple (e.g., three) exemplars simultaneously, and tests are conducted to determine if responding generalizes to untrained exemplars (Schroeder \& Baer, 1972). C-MET has the potential advantage of saving instructional time, as different exemplars are presented simultaneously during teaching (Panyan \& Hall, 1978). For example, Reeve, Reeve, Townsend, and Poulson (2007) measured acquisition and generalization when C-MET was arranged to teach helping behavior for four participants with ASD. The experimenters arranged teaching of four categories of helping (e.g., locating objects, putting items away, setting up an activity, carrying objects) and measured generalization to two novel categories (e.g., cleaning and replacing broken items) of helping (i.e., across-category generalization). Additionally, the experimenters arranged teaching of four exemplars (e.g., locating a ball, paint, puzzle piece, and pencil) within each category and measured generalization to one novel (e.g., locating a marble) exemplar (i.e., within-category generalization). All participants acquired the targeted helping responses, and demonstrated within- and acrosscategory generalized responding.

Previous research has demonstrated the effectiveness of S-MET and C-MET in the acquisition and generalization of different behaviors such as vocal imitation and social behavior (e.g., Eikeseth \& Nesset, 2003; Reeve et al., 2007). Studies have directly compared the effects of S-MET and C-MET. These comparison studies allow researchers to evaluate relative efficiency, and may provide helpful information to practitioners as to the training arrangement that is most beneficial for the consumers they serve. Previous studies directly compared the effects of S-MET and C-MET (e.g., Panyan \& Hall, 1978; Schroeder \& Baer, 1972; Schroeder, Schuster, \& Hemmeter, 1998; Wunderlich, Vollmer, Donaldson, \& Phillips, 2014). Overall, these studies have shown acquisition of targeted responses with both S-MET and C-MET, but more successful stimulus generalization during C-MET. For example, Wunderlich et al. (2014) evaluated the relative effectiveness (as measured by sessions to mastery) of S-MET and C-MET on the acquisition and generalization of tacts of letters or letter sounds for five participants with developmental disabilities. During S-MET, training occurred for one of three 
training exemplars. Training for a second exemplar occurred if generalization was not observed for the untrained serial exemplars. This procedure continued until all three exemplars had been trained or correct responding generalized to the untrained exemplars. During C-MET, the experimenters trained three exemplars simultaneously, and they evaluated generalization to one untrained exemplar. The results showed the C-MET condition required fewer sessions to mastery and resulted in a greater percentage of correct responses on concurrent generalization exemplars for all five participants.

One of the limitations of Wunderlich et al. (2014) is that number of training sessions may not be the best measure of efficiency, especially if there are differences in the number of exposures to stimuli or total training time across conditions (Black et al., 2016; Kodak et al., 2016; Yaw et al., 2014). It may be possible that the CMET condition required fewer training sessions, but that the total duration of these training sessions was greater than that of the S-MET condition. For example, shorter response latencies may be observed when the same target stimulus is repeatedly presented across trials (S-MET) in comparison to when varied stimuli are presented across trials (C-MET). Black et al. (2016) demonstrated that evaluating cumulative instructional sessions versus cumulative instructional time yielded different conclusions regarding instructional efficiency. Similarly, Yaw et al. (2014) suggested that mean training time per target was the most sensitive measure of efficiency. As such, comparison studies should include multiple measures before reaching firm conclusions regarding relative efficiency of specific procedures. Additionally, a consumer's previous instructional history may also affect the efficiency of a specific procedure (Coon \& Miguel, 2012; Freeman \& Lattal, 1992). Thus, it is possible that the efficiency of a specific MET procedure may also be affected by a participant's previous exposure to it.

Another procedure aimed at producing generalized responding is instructive feedback (IF) and involves the presentation of secondary targets during training trials. The primary targets are learner responses directly taught using prompts and consequences, whereas secondary targets are responses presented by the instructor without requiring any responses from the learner or delivering any consequences (Vladescu \& Kodak, 2013). These secondary targets have been presented prior to (e.g., Vladescu \& Kodak, 2013) or following (e.g., Reichow \& Wolery, 2011) primary targets. When presented prior to the primary target, the secondary target is presented before the delivery of the antecedent stimulus relevant to the primary target. For example, the instructor may present a picture of a plane and say, "This is a plane" (secondary target). The instructor then presents the antecedent stimulus, "What is it (while presenting a picture of a truck)?" If the learner responds with "truck" (primary target), the instructor delivers reinforcement. When presented following the training of primary targets, the secondary target is presented following the delivery of reinforcement for correct responses to the primary target. For example, the instructor delivered the antecedent, "What is it (while presenting a picture of a truck)?" The learner responds with "truck." The instructor delivers reinforcement and then presents the secondary target, a picture of a plane and the statement, "This is a plane." These arrangements have resulted the acquisition of secondary targets in the absence of direct reinforcement (e.g., Vladescu \& Kodak, 2013).

Recent studies have evaluated the usefulness of IF for improving instructional efficiency of learners with ASD (see Nottingham, Vladescu, \& Kodak, $\underline{\mathbf{2 0 1 5}}$ for a review of this literature). For example, Vladescu and Kodak (2013) assessed whether presenting secondary targets prior to the presentation of the primary target (antecedent portion of the trial) or following the consequence contingent upon the primary target (consequence portion of the trial) would affect skill acquisition. The authors also assessed whether participants would acquire the secondary targets during training of the primary targets. A comparison condition was added in which the participants were exposed to secondary targets in the absence of primary targets. The authors found that instruction was most efficient (least amount of training time) in the conditions in which secondary targets were incorporated. For three out of their four participants, secondary targets were acquired without direct 
consequence with no difference as to whether the secondary targets appeared during the antecedent or consequence portions of learning trials.

IF appears particularly amenable to programming for generalization, as instructors could teach primary targets and arrange different exemplars of these targets as secondary targets. In this case, instructors would present exemplars of the same target in the antecedent and consequence portion of training trials. Therefore, the purpose of the current study was to evaluate the effects and relative efficiency of S-MET and C-MET (similar to Wunderlich et al., 2014) on the acquisition of tacts by comparing the total number of training/probe sessions, exposures to mastery, and mean training time per mastered exemplar. Next, we evaluated whether these arrangements would produce generalization by arranging exemplars of the same target as secondary targets during training trials. To our knowledge, no previous study has evaluated presenting exemplars of primary targets as secondary targets to program for generalized responding.

\section{METHOD}

\section{Participants}

Participants were three children (Claire, Brandon, and Ralph) who had previously been diagnosed with ASD. They all received approximately $30 \mathrm{hr}$ per week of behavior analytic intervention that included the use of prompting and token reinforcement. Participants had been exposed to tact training using a mix of both S-MET and C-MET for at least 1 year. None of the participants had exposure to the presentation of secondary targets within learning trials.

Claire was an 8-year-old girl who had been receiving intervention for 4 years. She obtained a standard score of 55 (qualitative description: deficient to low) on the communication domain of the Battelle Developmental Inventory-Second Edition (BDI-2; Newborg, 2004). Claire performed all or nearly all skills through Level 2 on the mand, tact, listener, and intraverbal portions and all skills on the echoic subtest from the Verbal BehaviorMilestones Assessment and Placement Program (VB-MAPP; Sundberg, 2008).

Brandon was a 4-year-old boy who had been receiving intervention for 1 year. He obtained a standard score of 61 (low) on the communication domain of the BDI-2. Brandon performed all or nearly all skills though Level 3 on the mand, tact, listener, and intraverbal portions and all skills on the echoic subtest from the VB-MAPP.

Ralph was a 7-year-old boy who had been receiving intervention for 3 years. He obtained a standard score of 55 (deficient to low) on the communication domain of the BDI-2. Ralph performed all or nearly all skills though Level 3 on the mand, tact, listener, and intraverbal portions and all skills on the echoic subtest from the VBMAPP.

\section{Setting and Materials}

The study was conducted in an unattended, near-empty classroom (approximately $2.5 \mathrm{~m}$ by $3 \mathrm{~m}$ ) at the participants' school. The classroom contained a table and two chairs. Materials included paper data sheets, pencils, stimulus cards $(12.7 \mathrm{~cm}$ by $17.78 \mathrm{~cm})$ with pictures of unknown items on a white background, a digital timer, choice board, token board, and a video camera. The experimenter sat across the table from the participants (approximately . $6 \mathrm{~m}$ away), and a video camera was set up in the corner of the room to record sessions. Sessions began when the experimenter presented the initial antecedent stimulus and concluded after delivery of the last consequence of the last trial.

\section{Experimental Design, Measurement, and Interobserver Agreement}

We used an adapted alternating treatments design (Sindelar, Rosenberg, \& Wilson, 1985) to evaluate the relative efficacy of treatment conditions across the following dependent variables: unprompted and prompted 
correct and incorrect responses, and session duration. During all sessions, the experimenter recorded participants' unprompted and prompted correct and incorrect vocal responses in the presence of pictures. Only unprompted correct responses are displayed in the figures and were defined as vocally stating the name of the target prior to the delivery of a prompt. Unprompted incorrect responses were defined as errors of commission (engaging in a vocal response that is not the target response) and omission (engaging in no response) that occurred prior to the delivery of a prompt. Prompted correct responses were defined as imitating the experimenter's vocalization within $5 \mathrm{~s}$ of a vocal (echoic) prompt. Prompted incorrect responses were defined as errors of commission and omission that occurred after the delivery of a prompt. The experimenter started the timer prior to the first trial of the session, immediately before establishing attending by saying the participant's name, and stopped the timer immediately following the completion of the final component of the last trial to record session duration. Duration measures included the time participants spent consuming or engaging with putative reinforcers.

We calculated the total training and probe sessions to mastery by adding the number of training or probe sessions per condition. Exposures to mastery were calculated by adding the number of times per session that each primary target was presented (including training and error correction trials) until the mastery criterion was achieved. Total training time was calculated by adding session durations for each training session per condition. We calculated mean training time per mastered exemplar for each condition by dividing total training time of the session duration by the number of mastered exemplars (both training and generalization exemplars).

A second observer independently scored participants' responding from video recordings for a minimum of $33 \%$ of sessions across conditions. We calculated trial-by-trial interobserver agreement (IOA) by dividing the number of trials with an agreement by the total number of trials and converted the ratio to a percentage. An agreement in each trial was scored if the observer recorded the occurrence of the exact same dependent variables within the trial. Mean IOA for Claire was $96 \%$ (range, 89\%-100\%) during training conditions and 92\% (range, 89\%-100\%) during generalization and instructive feedback (IF) secondary target probes. For Claire's replication, mean IOA was $96 \%$ (range, 89\%-100\%) during training conditions and 90\% (range, 83\%-100\%) during generalization and IF secondary target probes. For Brandon, mean IOA was $94 \%$ (range, $83 \%-100 \%$ ) during training conditions and 95\% (range, 89\%-100\%) during generalization and IF secondary target probes. For Ralph, mean IOA was $91 \%$ (range, 83\%-100\%) during training conditions and 96\% (range, 83\%-100\%) during generalization and IF secondary target probes.

\section{Procedural Integrity and Procedural Integrity IOA}

An independent observer collected procedural integrity data for each participant during at least $33 \%$ of sessions across phases and conditions using a checklist of all correct experimenter behavior (specific experimenter behaviors for which we collected data are available from second author). We calculated procedural integrity by dividing the total number of correct experimenter behaviors by the number of correct experimenter behaviors plus the number of incorrect experimenter behaviors and multiplying by 100 . Mean procedural integrity scores were $100 \%$ for Claire during both initial and replication comparisons, 100\% for Brandon, and 99\% (range, 92\%$100 \%$ ) for Ralph. A second observer measured procedural integrity data during a minimum of $33 \%$ of sessions across participants. Trial-by-trial IOA was calculated by dividing the number of trials with an agreement on integrity by the total number of trials and converting to a percentage. Mean procedural integrity IOA was $100 \%$ for Claire during both the initial and replication comparisons, 99\% (range, 92\%-100\%) for Brandon, and 99\% (range, 92\%-100\%) for Ralph. 


\section{Preference Assessment and Token Economies}

The experimenter conducted separate tangible and edible multiple stimulus without replacement (MSWO; DeLeon \& Iwata, 1996) preference assessments with each participant. A picture of any item approached at least once was available on each participant's choice board.

Each session included a token board, as participants were familiar with the token economy as previously described. Prior to each session, participants selected a picture of an item from their choice board and placed it on their token board. After earning 10 tokens, the experimenter provided 2-min access to the preferred tangible or allowed $2 \mathrm{~min}$ to consume a preferred edible. All edibles were approximately the same size.

\section{Pretest and Stimuli}

We collected a pool of potential targets, which consisted of pictures of common objects, based on individual treatment goals and the New Jersey Core Curriculum Content Standards (State of New Jersey, 2014). For each potential target, we selected four exemplars such that the objects depicted in each picture differed along at least two dimensions (i.e., size, color, position of item). Next, we conducted three pretest trials for each potential target during which the experimenter said, "What is it?" and allowed the participant $5 \mathrm{~s}$ to respond. No feedback was provided for correct or incorrect responses. A token and praise (e.g., "Great job working”) were provided for appropriate sitting or attending behaviors during the intertrial interval (ITI), about every other trial. If a participant responded correctly to a potential target by tacting either the target name or any features of the target at least once, that stimulus was discarded and replaced.

We assigned four exemplars of three targets ( 4 exemplars times 3 targets equals 12 total targets for each condition) to one of four conditions: (a) the S-MET condition, (b) the C-MET condition, (c) the IF condition, and (d) the control condition. We used the logistical analysis method (Wolery, Gast, \& Ledford, 2014) to assign target sets across conditions. That is, we based assignment on the number of syllables in the target responses to equate syllables across stimulus sets, and targets that were phonetically or visually similar were not included within the same set. See Table $\underline{1}$ for a list of targets for each participant.

Table 1. Training Sets for All Conditions

\begin{tabular}{|l|l|l|l|l|l|}
\hline Participant & $\begin{array}{l}\text { Serial MET } \\
\text { Condition }\end{array}$ & $\begin{array}{l}\text { Concurrent MET } \\
\text { Condition }\end{array}$ & $\begin{array}{l}\text { IF } \\
\text { Condition }\end{array}$ & Control 1 & Control 2 \\
\hline Claire & Badge & Dough & Wrench & Ax & Wok \\
\hline & Chalkboard & Fountain & Oyster & Necklace & Ice Cube \\
\hline $\begin{array}{l}\text { Claire } \\
\text { (replication) }\end{array}$ & Artichoke & Grasshopper & Suspenders & Celery & Podium \\
\hline & Hydrant & Squid & Vest & Rug & Squid \\
\hline & Cantaloupe & Radio & Chimney & Donkey & Ruler \\
\hline Brandon & Wok & Wrench & Envelope & Computer & Fireman \\
\hline & & & Badge & Ax & Dough \\
\hline
\end{tabular}




\begin{tabular}{|l|l|l|l|l|l|}
\hline & Ginger & Podium & Fountain & Screwdriver & Oyster \\
\hline & Suspenders & Chalkboard & Artichoke & Suitcase & Grasshopper \\
\hline Ralph & Branch & & Vest & Rug & Dough \\
& & & & & \\
\hline & Tweezer & Ruler & Oyster & Ginger & Artichoke \\
\hline & Podium & Envelope & Celery & Suspenders & Fountain \\
\hline
\end{tabular}

Note. MET: multiple exemplar training: IF: instructional feedback

\section{Procedure}

Baseline and training sessions consisted of nine trials. Prior to each session, we determined the order that targets were presented across trials such that no target was presented on more than two consecutive trials. Prior to each IF session, we separately determined the order of the antecedent secondary targets, the primary targets, and the consequence secondary targets such that secondary targets were not presented with the same primary targets across sessions.

Conditions were conducted one to three times per day, one to three days per week, depending on participants' availability. Training in a condition continued until participants demonstrated $100 \%$ correct unprompted responses for two consecutive sessions (from the presentation of the initial antecedent stimuli to the delivery of the consequence of the last trial).

\section{Baseline and control}

The experimenter established attending behavior (participant was sitting still with his hands in lap) by saying the participant's name and modeling the behavior, if necessary. The experimenter presented the picture, said, "What is it?" and waited up to $5 \mathrm{~s}$ for the participant to respond. No feedback was provided for unprompted correct or incorrect responses. The experimenter presented the next trial after a 3 to $5 \mathrm{~s}$ ITI. Appropriate session behavior (e.g., sitting with hands down) was followed by praise and a token during the ITI, approximately every other trial. The purpose of the control condition was to detect maturation and history effects. This condition was terminated at the end of training for all participants.

\section{Training}

The first two instructional sessions for the S-MET, C-MET, and IF conditions included trials in which the experimenter provided an echoic prompt immediately following the presentation of the antecedent stimuli (i.e., 0 -s prompt delay [PD]). Following two consecutive sessions with $100 \%$ prompted correct responding at a $0-s$ PD, the experimenter increased the PD to $5 \mathrm{~s}$. During trials conducted with the 5-s PD, the experimenter presented the antecedent stimuli and waited up to $5 \mathrm{~s}$ for the participant to respond. The experimenter delivered a token and praise following prompted correct (for 0-s PD only) and unprompted (5-s PD) correct responses. Following prompted (0-s PD) and unprompted (5-s PD) incorrect responses, the experimenter conducted error-correction (EC) during which the trial was re-presented until the participant engaged in a prompted (0-s PD) or unprompted (5-s PD) correct response (i.e., re-present until independent EC procedure; Carroll, Joachim, St. Peter, \& Robinson, 2015). Responses that occurred during EC were not included in the figures. 
Serial multiple exemplar training

For each of the three targets assigned to the S-MET condition, one exemplar was randomly selected to serve as the training target, and the three remaining exemplars were reserved to test for generalization. During each session, one exemplar of each of the three training targets was presented three times for a total of nine trials. During each trial the experimenter held up a picture, said, "What is it?", provided a prompt, implemented EC if necessary, and delivered reinforcement according to the procedures described above.

\section{Concurrent multiple exemplar training}

For each of the three targets assigned to the C-MET condition, three exemplars were randomly selected to serve as training targets, and one exemplar was used to test for generalization. For each session, three exemplars of each training target were presented once per session for a total of nine trials. During each trial, the experimenter held up a picture, said "What is it?", provided a prompt, implemented EC if necessary, and delivered reinforcement according to the procedures described above.

Instructive feedback

For each of the three targets assigned to the IF condition, one exemplar of each target was selected to serve as the training target. A second exemplar of each target was selected to serve as the secondary target for the antecedent portion of trials, and a third exemplar was selected to serve as the secondary target during the consequence portion of trials. Lastly, a fourth exemplar was reserved to test for generalization. Each session consisted of nine trials. Each trial consisted of the experimenter consecutively presenting a secondary target/exemplar, the primary target, and another secondary target/exemplar (the specific arrangement of primary and secondary targets for each trial varied across sessions as described above). In other words, each trial began with the experimenter presenting a secondary target for $3 \mathrm{~s}$ (e.g., held up a picture of an artichoke, and said, "This is an artichoke"). Next, the experimenter presented the antecedent stimulus relevant to a primary target (e.g., held up a picture of a badge and said, "What is it?"). Primary targets were trained using the procedure described above. Following the delivery of reinforcement for prompted (0-S PD) correct and unprompted (5-S PD) correct responses relevant to the primary target, the experimenter presented another secondary target for $3 \mathrm{~s}$ (e.g., held up a picture of fountain, and said "this is a fountain"). Participants were not required to respond following the presentation of a secondary target, and no programmed consequences were provided contingent upon responses.

\section{Generalization and Secondary Target Probes}

All generalization probe sessions were conducted immediately prior to training sessions using procedures described in baseline. Secondary target probes were conducted immediately prior to instructional sessions to evaluate the acquisition of secondary targets while primary targets were undergoing training. These probe sessions were conducted using procedures described in baseline.

\section{RESULTS}

We compared the relative efficiency of the S-MET, C-MET, and IF conditions by assessing total number of training/probe sessions, exposures to mastery, total training time, and mean training time per mastered exemplar. During her first comparison, Claire required seven, six, and five training sessions to master training exemplars in the S-MET, C-MET, and IF conditions, with 69, 56, and 31 exposures to mastery, respectively (Figure 1). Although the S-MET condition required the largest number of training sessions, it was associated with the lowest total training time (13 $\mathrm{min} 23 \mathrm{~s}$ ) and lowest mean training time per mastered exemplar ( $1 \mathrm{~min} 7 \mathrm{~s}$ ). Claire mastered all secondary targets and generalization exemplars across conditions without direct training (Figure 2). 

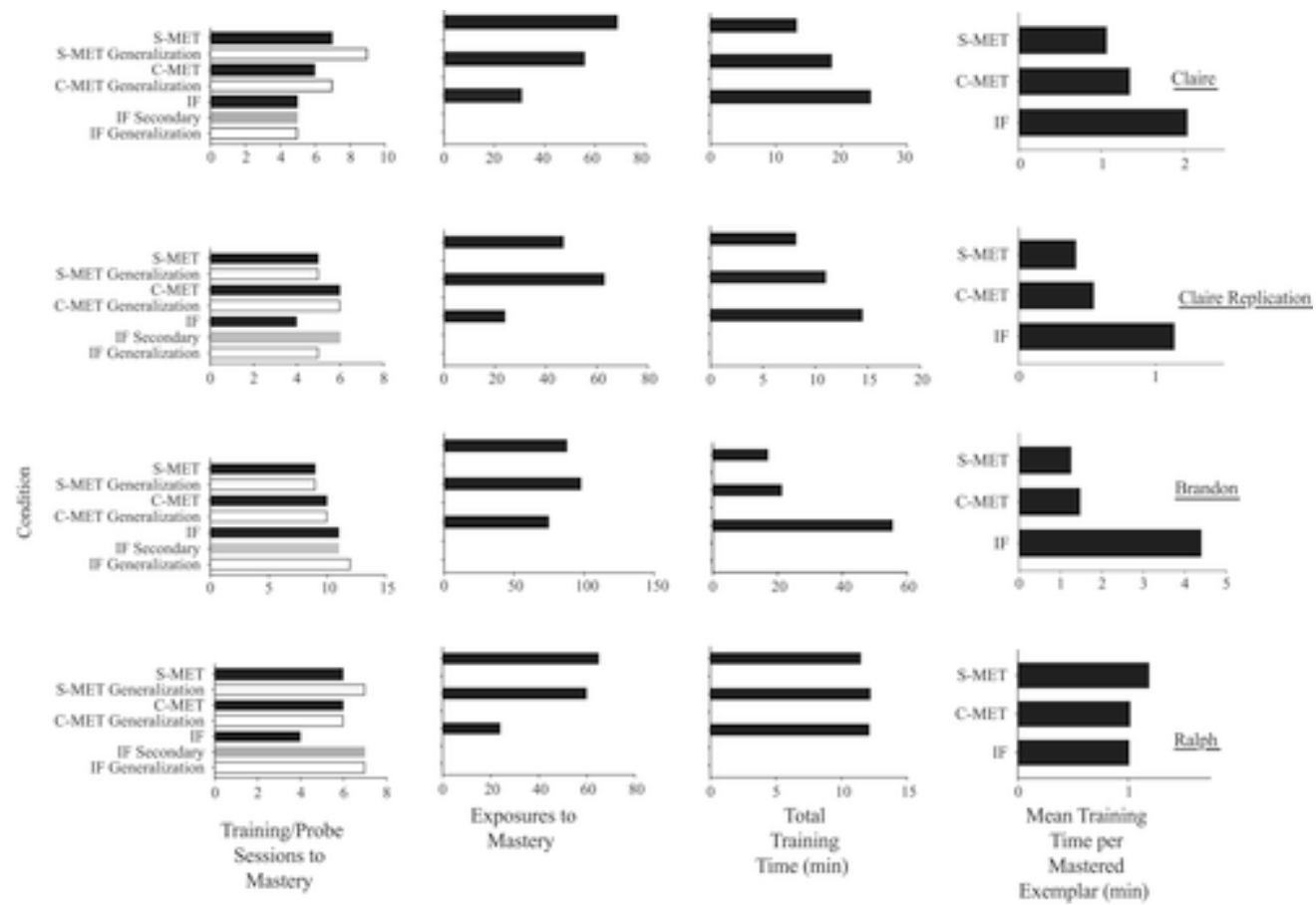

The number of training/probe sessions to mastery, exposures to mastery, total training time and mean training time per mastered exemplar for Claire, Claire's replication, Brandon, and Ralph across the serial, concurrent and instructive feedback conditions.

Training Targets
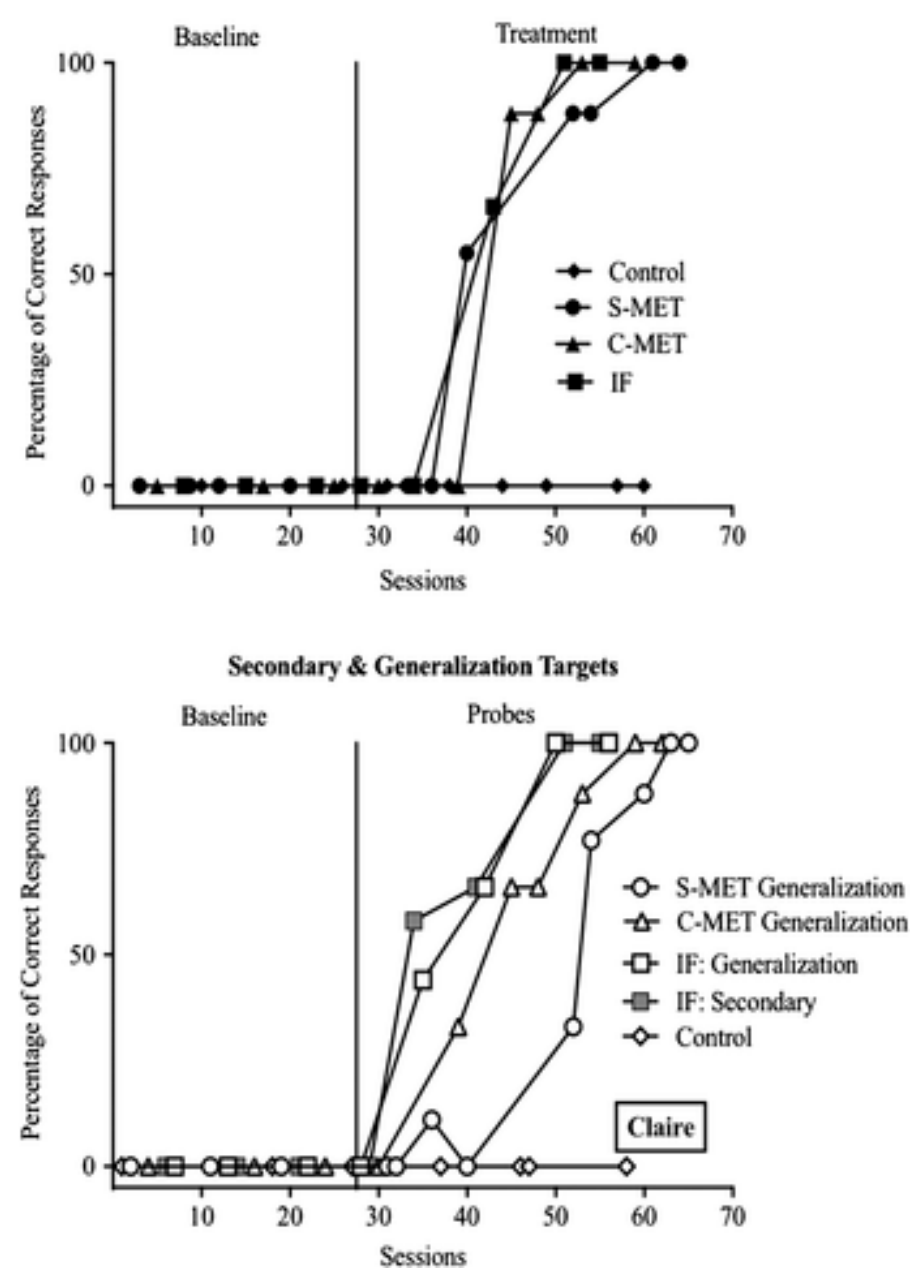
The percentage of correct responses to training targets (top panel) and secondary and generalization targets (bottom panel) in each condition for Claire.

During Claire's replication, she required five, six, and four training sessions to master training exemplars in the SMET, C-MET, and IF conditions, with an overall of 47, 63, and 24 exposures to mastery respectively (Figure 1). The S-MET condition was again associated with the lowest total training time ( 8 min $19 \mathrm{~s}$ ) and lowest mean training time per mastered exemplar (42 s). Like her initial evaluation, Claire mastered all secondary targets and generalization exemplars without direct training (Figure $\underline{3}$ ).
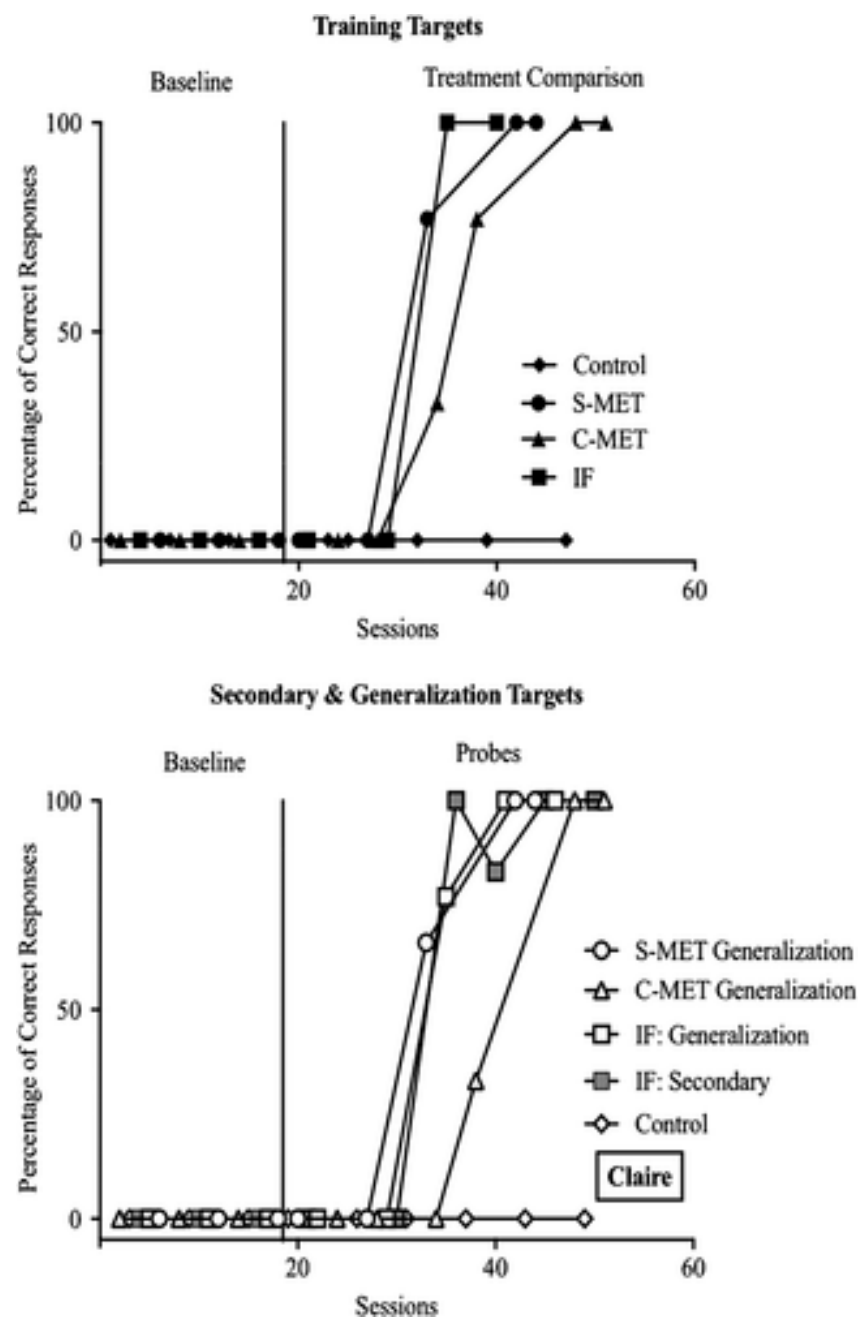

The percentage of correct responses to training targets (top panel) and secondary and generalization targets (bottom panel) in each condition of the replication phase for Claire.

Brandon required the least number of training sessions, lowest total training time, and lowest mean training time per mastered exemplar in the S-MET condition as well (Figure 1). More specifically, he required 9, 10, and 11 training sessions to master training exemplars in the S-MET, C-MET, and IF conditions, with 88, 98, and 75 exposures to mastery, respectively. The S-MET condition was associated with the lowest total training time (17 min $10 \mathrm{~s}$ ) and lowest mean training time per mastered exemplar (1 min $26 \mathrm{~s}$ ). Brandon demonstrated mastery of all secondary targets and generalization exemplars without direct training (Figure 4 ). 


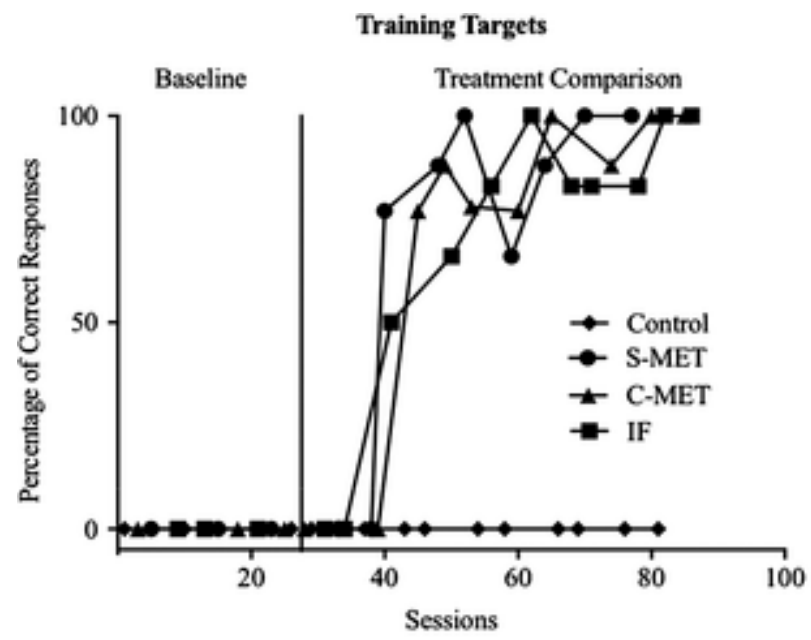

Secondary \& Generalization Targets

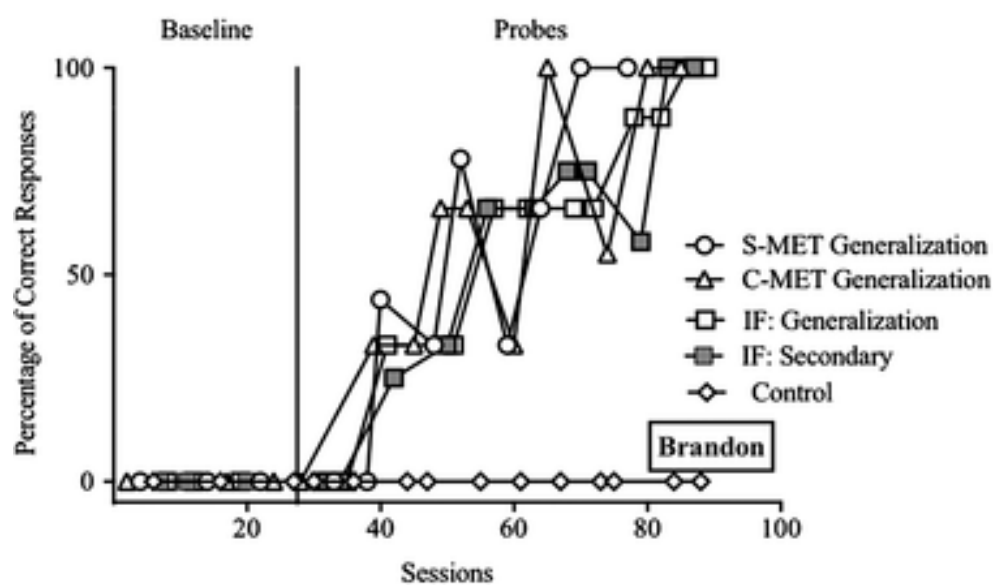

The percentage of correct responses to training targets (top panel) and secondary and generalization targets (bottom panel) in each condition for Brandon.

Ralph required six, six, and four training sessions to master training exemplars in the S-MET, C-MET, and IF conditions, with 65, 60, and 24 exposures to mastery, respectively (Figure $\underline{1}$ ). He mastered all secondary targets and generalization exemplars in the C-MET and IF conditions (Figure $\underline{5}$ ). He demonstrated mastery of generalization exemplars in the S-MET condition for two of the three targets. We were unable to continue the evaluation with Ralph due to the end of the school semester. Thus, serial training was the only condition that did not lead to mastery level responding for all generalization targets. Ralph's S-MET condition was associated with the lowest total training time (11 min $48 \mathrm{~s}$ ) even though not all generalization exemplars were acquired; however, the IF condition was associated with the lowest mean training time per mastered exemplar (1 min $1 \mathrm{~s}$; Figure 1). 

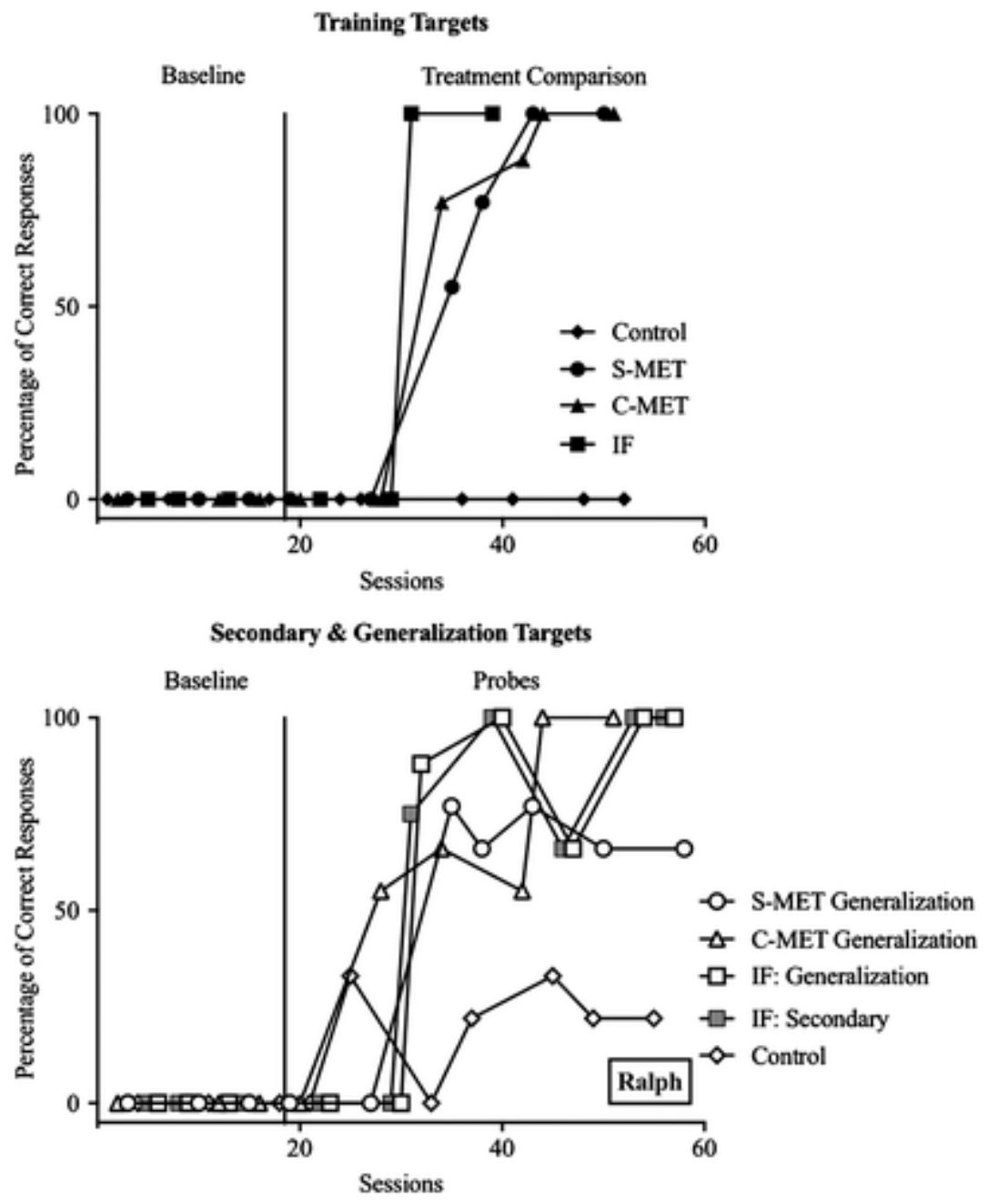

The percentage of correct responses to training targets (top panel) and secondary and generalization targets (bottom panel) in each condition for Ralph.

Responding remained low or at zero for all participants during the control conditions (Figures $\underline{\mathbf{2}} \underline{-5}$ ).

\section{DISCUSSION}

Our results showed that all training conditions (i.e., S-MET, C-MET, and IF) produced acquisition of training exemplars and generalization to nontrained exemplars. However, the S-MET condition resulted in the lowest mean training time per mastered exemplar for two participants, whereas the IF condition was the most efficient for the third. Although seemingly small differences in total training time and mean training time per mastered exemplar may be apparent for some participants, we consider even small differences to be of potential clinical significance. It is easy to imagine how these small differences could accumulate to become more substantial over the long periods of intensive and comprehensive behavioral intervention that is likely to be implemented over the course of years for consumers with ASD.

The finding that S-MET was more efficient, even for a subset of participants, contrasts with previous comparative studies that have generally demonstrated the superiority of C-MET (e.g., Wunderlich et al., 2014). In fact, to our knowledge, this is the first study to demonstrate that S-MET resulted in more efficient acquisition and stimulus generalization relative to C-MET. For this reason, the study warrants additional replication. This finding suggests that it may not always be necessary to arrange concurrent training of exemplars when teaching 
individuals with ASD as previous studies have suggested. It is important to note that we evaluated mean training time per mastered exemplar to draw a conclusion as to the relative efficiency of the teaching conditions, whereas previous studies did not. Using this type of measurement allowed us to evaluate relative efficiency in a way that may have differed if data were examined using a different measure. For example, the results of Claire's initial evaluation indicated that S-MET was the least efficient condition based on training sessions to mastery, as the S-MET condition was associated with the most training sessions. However, when evaluating instructional time, the S-MET condition required the lowest total and mean training time per mastered exemplar, and the CMET and IF conditions were associated with total instructional time that was approximately $42 \%$ and $86 \%$ greater, respectively. Such findings suggest that relative efficiency of interventions may vary as a function of how it is measured. Future studies should evaluate the generality of these findings and include measures such as duration of sessions, mean training time per mastered stimulus, and total training and probe sessions to mastery, to ensure accurate conclusions regarding relative efficiency are reached.

The results of the current evaluation suggest that under certain conditions, concurrent training of exemplars may impede instruction, and result in additional instructional time. This may be the case due to the increased number of discriminations required during C-MET sessions. More specifically, it seems that the S-MET was relatively easier as each training session involved three unique discriminations (each of which occurred three times per session), whereas nine unique discriminations (each of which occurred one time per session) were required during C-MET sessions. The increased number of discriminations may have resulted in increased total training time. Additionally, in the S-MET, stimulus control over a response was established during training involving a single exemplar, whereas in the C-MET, stimulus control over a response was established during training involving multiple exemplars. We chose to arrange conditions in this manner as they mirror how these conditions would have likely been implemented clinically and how they have been arranged in previous studies (e.g., Wunderlich et al., 2014). Future studies could consider the number and types of discriminations in their procedural preparations.

The results of the current evaluation are somewhat contrary to previous IF studies. In previous studies, conditions incorporating secondary targets have consistently been associated with the most efficient instruction (e.g., Reichow \& Wolery, 2011; Vladescu \& Kodak, 2013; Wolery, Schuster, \& Collins, 2000). Although the IF condition was most efficient for Ralph, this condition led to the longest mean training time per mastered exemplar and overall training time for Claire and Brandon. The current study included two secondary targets per trial (one in the antecedent portion of each trial and another in the consequence portion of each trial). This differs from most IF studies that have typically arranged the presentation of one secondary target in either the antecedent or consequence portion of training trials (e.g., Cromer, Schuster, Collins, \& Grisham-Brown, 1998; Vladescu \& Kodak, 2013; Wolery, Ault, Gast, Doyle, \& Mills, 1990). Nottingham, Vladescu, Kodak, and Kisamore (2017) included two conditions that involved the presentation of two secondary targets during each trial. Across three comparisons, a condition involving multiple targets was most efficient twice. Future research is needed to evaluate what effect multiple secondary target presentations have on training efficiency and whether other arrangements (e.g., presenting one secondary target per trial, or presenting two secondary targets during the consequence portion of trials) influence acquisition and generalization.

S-MET did not lead to generalization of all stimuli with one participant (Ralph). If additional training had been necessary to produce mastery of all targets, then an increase in overall training time would have been observed, rendering S-MET less efficient. Future research should investigate what effect additional training would have on the efficiency of S-MET. It could be possible that Ralph's history of instruction played a role in the acquisition of targets under S-MET. Perhaps he had more exposure to C-MET arrangements and therefore did not acquire all targets across conditions. 
The findings from the current and previous studies raise interesting questions as to how practitioners should select a teaching arrangement that most efficiently produces acquisition and generalized outcomes. Previous research has shown that the most efficient instructional arrangement is often learner-specific (e.g., Boudreau, Vladescu, Kodak, Argott, \& Kisamore, 2015; Koehler, Iwata, Roscoe, Rolider, \& O'Steen, 2005). If this is the case, future research should attempt to delineate the variables that influence learner-specific response patterns. The current study included three participants with extensive tact repertoires. Future studies could replicate the current procedures with additional participants with more varied verbal skills. We arranged training of three exemplars per target in the C-MET condition and exposure to three exemplars (direct training of one exemplar and exposure to two exemplars as secondary targets) in the IF condition. Perhaps training or exposing participants to fewer exemplars (e.g., two) may have resulted in different outcomes. Relatedly, it may be worthwhile to explore the development of an assessment to identify the optimal number of exemplars needed to produce the most efficient acquisition and generalized responding similar to prior studies that have examined optimal teaching arrangements for learners (e.g., McGhan \& Lerman, 2013; Seaver \& Bourret, 2014).

It also possible that the most efficient arrangement is task specific. In the current study, and in previous research (e.g., Cuvo et al., 1980; Schroeder et al., 1998; Wunderlich et al., 2014), we evaluated acquisition and generalization of tacts. Perhaps we would have observed different patterns of responding if we had targeted more complex verbal (e.g., intraverbals) or prosocial behavior. In such cases, training multiple exemplars concurrently may be necessary. This may be because more complex stimuli may share fewer characteristics that are similar or may be presented in a variety of stimulus contexts, therefore requiring the presentation of multiple exemplars for generalization. For example, when teaching participants to offer help or assistance, the antecedents under which the response occurs may share fewer stimulus features (e.g., dropping materials and requiring assistance to clean up, opening a door when hands are full with items, cleaning up a spilled mess). Therefore, additional exemplars may be needed to establish the desired stimulus control. It could be hypothesized that any physical similarities across teaching targets used in the current study (picture cards) had an effect on participants' generalized responses across stimuli. Future research is needed to identify the conditions under which targeting concurrent exemplars is more beneficial than serial presentation and whether decisions regarding MET need to be made considering learner-specific characteristics, the type of skill targeted, or both.

Our overall results indicated that the S-MET condition produced efficient acquisition and generalization for two of three participants. Additional research is needed to identify the conditions under which S-MET, C-MET, and IF would be most successful for learners. Outcomes of these studies will help guide clinicians to arrange the most effective instructional practices that lead to generalization for individuals with ASD.

\section{References}

Baer, D. M., Wolf, M. M., \& Risley, T. R. (1968). Some current dimensions of applied behavior analysis. Journal of Applied Behavior Analysis, 1, 91- 97. https://doi.org/10.1901/jaba.1968.1-91

Black, M. P., Skinner, C. H., Forbes, B. E., McCurdy, M., Coleman, M. B., Davis, K., \& Gettelfinger, M. (2016). Cumulative instructional time and relative effectiveness conclusions: Extending research on response intervals, learning, and measurement scale. Behavior Analysis in Practice, 9, 58- 62. https://doi.org/10.1007/240617-016-0114-3

Boudreau, B. A., Vladescu, J. C., Kodak, T. M., Argott, P. J., \& Kisamore, A. N. (2015). A comparison of differential reinforcement procedures with children with autism. Journal of Applied Behavior

Analysis, 48, 918- 923. https://doi.org/10.1002/jaba.232 
Carroll, R. A., Joachim, B. T., St. Peter, C. C., \& Robinson, N. (2015). A comparison of error-correction procedures on skill acquisition during discrete-trial instruction. Journal of Applied Behavior

Analysis, 48, 1- 17. https://doi.org/10.1002/jaba.205

Catania, C. A. (2007). Learning, $4^{\text {th }}$ ed. Cornwall-on-Hudson, NY: Sloan Publishing.

Coon, J. T., \& Miguel, C. F. (2012). The role of increased exposure to transfer-of-stimulus-control procedures on the acquisition of intraverbal behavior. Journal of Applied Behavior Analysis, 45, 657- 666. https://doi.org/10.1901/jaba.2012.45-6

Cromer, K., Schuster, J. W., Collins, B. C., \& Grisham-Brown, J. (1998). Teaching information on medical prescriptions using two instructive feedback schedules. Journal of Behavioral Education, 8, 37- 61. https://doi.org/10.1023/A: 1022812723663

Cuvo, A. J., Klevans, L., Borakove, S., Borakove, L. S., Van Landuyt, J., \& Lutzker, J. R. (1980). A comparison of three strategies for teaching object names. Journal of Applied Behavior Analysis, 13, 249- 257. https://doi.org/10.1901/jaba.1980.13-249

DeLeon, I. G., \& Iwata, B. A. (1996). Evaluation of a multiple-stimulus presentation format for assessing reinforcer preference. Journal of Applied Behavior Analysis, 29, 519- 532. https://doi.org/10.1901/jaba.1996.29-519

Eikeseth, S., \& Nesset, R. (2003). Behavioral treatment of children with phonological disorder: The efficacy of vocal imitation and sufficient-response-exemplar training. Journal of Applied Behavior Analysis, 36, 325- 337. https://doi.org/10.1901/jaba.2003.36-325

Freeman, T. J., \& Lattal, K. A. (1992). Stimulus control of behavioral history. Journal of the Experimental Analysis of Behavior, 57, 5- 15. https://doi.org/10.1901/jeab.1992.57-5

Kodak, T., Campbell, V., Bergmann, S., LeBlanc, B., Kurtz-Nelson, E., Cariveau, T., . . Mahon, J. (2016). Examination of efficacious, efficient, and socially valid error-correction procedures to teach sight words and prepositions to children with autism spectrum disorder. Journal of Applied Behavior Analysis, 49, 532- 547. https://doi.org/10.1002/jaba.310

Koehler, L. J., Iwata, B. A., Roscoe, E. M., Rolider, N. U., \& O'Steen, L. E. (2005). Effects of stimulus variation on the reinforcing capability of nonpreferred stimuli. Journal of Applied Behavior Analysis, 38, 469- 484. https://doi.org/10.1901/jaba.2005.102-04

McGhan, A. C., \& Lerman, D. C. (2013). An assessment of error-correction procedures for learners with autism. Journal of Applied Behavior Analysis, 46, 626- 639. https://doi.org/10.1002/jaba.65

Newborg, J. (2004). Batelle Developmental Inventory, $2^{\text {nd }}$ ed. (BDI-2). MA Boston: Houghton Mifflin Harcourt.

Nottingham, C. L., Vladescu, J. C., \& Kodak, T. (2015). Incorporating additional targets into learning trials for individuals with autism spectrum disorder. Journal of Applied Behavior Analysis, 48, 227- 232. https://doi.org/10.1002/jaba.179

Nottingham, C. L., Vladescu, J. C., Kodak, T., \& Kisamore, A. N. (2017). Incorporating multiple secondary targets into learning trials for individuals with autism spectrum disorder. Journal of Applied Behavior Analysis, 50, 653- 661. https://doi.org/10.1002/jaba.396

Panyan, M. C., \& Hall, R. V. (1978). Effects of serial versus concurrent task sequencing on acquisition, maintenance, and generalization. Journal of Applied Behavior Analysis, 11, 67- 74. https://doi.org/10.1901/iaba.1978.11$\underline{67}$ 
Reeve, S. S., Reeve, K. F., Townsend, D. B., \& Poulson, C. L. (2007). Establishing a generalized repertoire of helping behavior in children with autism. Journal of Applied Behavior

Analysis, 40, 123- 136. https://doi.org/10.1901/jaba.2007.11-05

Reichow, B., \& Wolery, M. (2011). Comparison of progressive prompt delay with and without instructive feedback. Journal of Applied Behavior Analysis, 44, 327- 340. https://doi.org/10.1901/jaba.2011.44-327

Rimland, B. (1964). Infantile autism: The syndrome and its implications for a neural theory of behavior. New York, NY: Appleton Century Croft.

Schroeder, C. M., Schuster, J. W., \& Hemmeter, M. L. (1998). Efficiency in programming for generalization: Comparison of two methods for teaching expressive labeling to preschoolers with developmental delays. Journal of Developmental and Physical

Disabilities, 10, 109- 131. https://doi.org/10.1023/A:10228023631613

Schroeder, G. L., \& Baer, D. M. (1972). Effects of concurrent and serial training on generalized vocal imitation in retarded children. Developmental Psychology, 6, 293- 301. https://doi.org/10.1037/h0032121

Seaver, J. L., \& Bourret, J. C. (2014). Evaluation of response prompts for teaching behavior chains. Journal of Applied Behavior Analysis, 47, 777- 792. https://doi.org/10.1002/jaba.159

Sindelar, P. T., Rosenberg, M. S., \& Wilson, R. J. (1985). An adapted alternating treatments design for instructional research. Education and Treatment of Children, 8, 67- 76.

State of New Jersey (2014). New Jersey Core Curriculum Content Standards. Retrieved April 6, 2016, from State of New Jersey, Department of Education website: http://www.state.nj.us/education/cccs/

Stokes, T. F., \& Baer, D. M. (1977). An implicit technology of generalization. Journal of Applied Behavior Analysis, 10, 349- 367. https://doi.org/10.1901/jaba.1977.10-349

Sundberg, M. L. (2008). Verbal behavior milestones assessment and placement program: The VP-MAPP. Concord, CA: AVB Press.

Vladescu, J. C., \& Kodak, T. M. (2013). Increasing instructional efficiency by presenting additional stimuli in learning trials for children with autism spectrum disorders. Journal of Applied Behavior Analysis, 46, 805- 816. https://doi.org/10.1002/jaba.70

Wolery, M., Ault, M. J., Gast, D. L., Doyle, P. M., \& Mills, B. (1990). Use of choral and individual attentional responses with constant time delay when teaching sign word reading. Remedial and Special Education, 11, 47- 58.

Wolery, M., Gast, D. L., \& Ledford, J. R. (2014). Comparison designs. In D. L. Gast \& J. R. Ledford (Eds.), Single case research methodology: Applications in special education and behavioral sciences (pp. 297-345). New York, NY: Routledge.

Wolery, T. D., Schuster, J. W., \& Collins, B. C. (2000). Effects on future learning of presenting non-target stimuli in antecedent and consequent conditions. Journal of Behavioral Education, 10, 77- 94. https://doi.org/10.1023/A:1016679928480

Wunderlich, K. L., Vollmer, T. R., Donaldson, J. M., \& Phillips, C. L. (2014). Effects of serial and concurrent training on acquisition and generalization, Journal of Applied Behavior Analysis, 47, 723- 737. https://doi.org/10.1002/iaba.154 
Yaw, J., Skinner, C. H., Skinner, A. L., Maurer, K., Cihak, D., Wilhoit, B., \& Booher, J. (2014). Measurement scale influences in the evaluation of sight-word reading interventions. Journal of Applied Behavior Analysis, 47, 360- 379. https://doi.org/10.1002/jaba.126 